\title{
Mechanical Properties of Silk: Interplay of Deformation on Macroscopic and Molecular Length Scales
}

\author{
Igor Krasnov, ${ }^{1}$ Imke Diddens, ${ }^{1}$ Nadine Hauptmann, ${ }^{1}$ Gesa Helms, ${ }^{1}$ Malte Ogurreck, ${ }^{1}$ Tilo Seydel, ${ }^{2}$ \\ Sérgio S. Funari, ${ }^{3}$ and Martin Müller ${ }^{1, *}$ \\ ${ }^{1}$ Institut für Experimentelle und Angewandte Physik der Christian-Albrechts-Universität zu Kiel, D-24098 Kiel, Germany \\ ${ }^{2}$ Institut Laue-Langevin, 6, rue Jules Horowitz, BP 156, F-38042 Grenoble Cedex 9, France \\ ${ }^{3}$ HASYLAB, DESY, Notkestrasse 85, D-22603 Hamburg, Germany
}

(Received 21 December 2006; published 31 January 2008)

\begin{abstract}
Using an in situ combination of tensile tests and x-ray diffraction, we have determined the mechanical properties of both the crystalline and the disordered phase of the biological nanocomposite silk by adapting a model from linear viscoelastic theory to the semicrystalline morphology of silk. We observe a strong interplay between morphology and mechanical properties. Silk's high extensibility results principally from the disordered phase; however, the crystals are also elastically deformed.
\end{abstract}

Natural silks exhibit extraordinary mechanical properties, combining high tensile strength with a high elongation at failure. Producing man-made fibers with such properties requires a considerable input of energy compared to natural silk fibers spun from aqueous protein solution at ambient temperature. The toughest silk known is spun by spiders. However, it has recently been shown that, chemically and rheologically closely related, silkworm silk [1] could reach comparable parameters in an optimized spinning process [2].

The similarity between spider and silkworm silk fibers extends to their morphology. Both are semicrystalline nanocomposites, with ordered regions ( $\beta$-sheet protein nanocrystals) embedded in a softer matrix of disordered material (spider silk, [3]; silkworm fibroin, [4]). Tensile deformation of silk fibers involves a strong contribution of viscoelastic material, presumably in the disordered regions [5]. However, detailed information on the relaxation mechanism is still missing.

The mesoscopic structure of polymer nanocomposites is the key to their mechanical properties [6]. Mimicking nature's spinning process to produce artificial fibers with optimized morphology and, thus, optimal mechanical performance either from silkworm or recombinant spider silk spinning dope [7] would be highly desirable. The models of silk available today do not directly connect structure and macroscopic properties. Termonia's model [8] describes silk as a hydrogen-bonded rubberlike matrix with embedded stiff crystals serving as cross-link sites. At their surface, another ordered phase of protein molecules is required in order to explain the static mechanical properties. The assumed high rigidity of the $\beta$-sheet crystals has been questioned by x-ray diffraction results [9]. While the recent model by Porter et al. [10] and Vollrath and Porter [11] includes morphological parameters such as ordered/ disordered fractions, which are very difficult to obtain experimentally, deformation processes in crystals and ma- trix are neglected. A molecular model for spider capture silk is available but restricted to this very specialized silk type [12].

The aim of this Letter is to establish a model for silk incorporating the macroscopic mechanical behavior, in particular, viscoelasticity, on the basis of its semicrystalline morphology. To this end, we combined high-resolution cyclic stress-strain measurements of single silkworm silk fibers (such as reported in [13-15]) with in situ tensile tests during synchrotron radiation x-ray diffraction experiments (see previous work $[3,9,16]$ ), the latter probing directly the deformation of the nanocrystals.

Silkworm silk [17] was investigated at ambient humidity content in all experiments. The stretching measurements on single fibers were carried out with a piezostretching device equipped with a force sensor [18]. Force and distance were sampled with $1000 \mathrm{~s}^{-1}$. The triangular strain excitation signal shown in Fig. 1(a) (right-hand scale) is a stepwise realization of a constant rate of strain change $(0.1 \% / \mathrm{s})$. The stress-response function was measured for increasing and decreasing strain on a previously unstretched fiber ( $2.0 \mathrm{~mm}$ initial length) and then again in a second cycle for the same, thus, prestretched sample.

$\mathrm{X}$-ray diffraction experiments were carried out in situ during a stretching experiment (strain rate 0.025\%/s [19], $4.1 \mathrm{~mm}$ initial length) on six fiber bundles of silkworm silk [17] required for a good signal-to-noise ratio. The computer-controlled tensile testing device consisted of a $\mathrm{dc}$ motor and a force sensor. Synchrotron radiation $\mathrm{x}$ rays (beam size $200 \mu \mathrm{m} \times 200 \mu \mathrm{m}$ ) were used at beam line A2, HASYLAB (Hamburg, Germany). Fiber diffraction patterns were recorded on a two-dimensional CCD detector. The lattice strain of the $\beta$-sheet fibroin crystals in the direction of the tensile stress was determined from the shift of the radial position of the 002 reflection $[4,9,20]$.

The results of the mechanical measurements on a single silk fiber are shown in Fig. 1. In all three graphs, curves (i) 

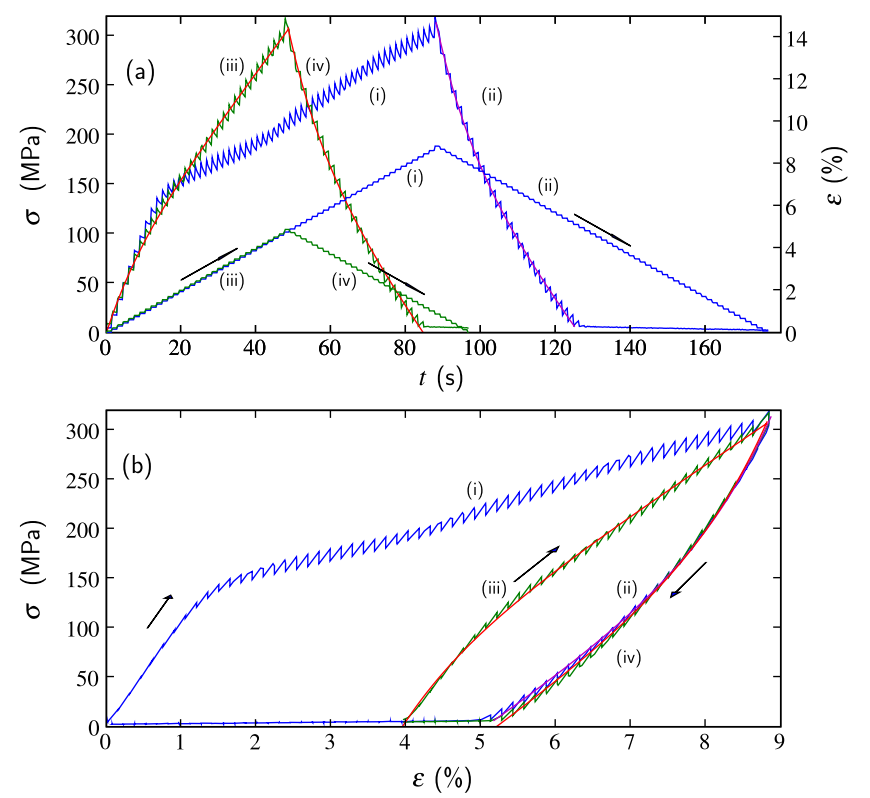

FIG. 1 (color online). Cyclic tensile stretching measurements on a single silkworm silk fiber. In the graphs (i), (ii) denote the fiber in the initial state (first stretching cycle) and (iii), (iv) in the prestretched state (second cycle), respectively. (i), (iii) stand for increasing and (ii), (iv) for decreasing strain. Smooth curves are fits with a linear viscoelastic model (see text and Fig. 2). (a) (bottom, right-hand scale) Stepwise realization of a triangular strain excitation curve (two slopes), with strain rate $\dot{\varepsilon}_{0}=0.1 \%$ per second. (a) (left-hand scale) Time dependence of the stress response to the strain excitation. (b) Stress-strain curves as obtained from (a).

and (ii) denote data from the initially unstretched sample, (iii) and (iv) from the same sample, which was initially stretched with a strain amplitude $\varepsilon \approx 9 \%$. (i) and (iii) were measured with increasing strain and (ii) and (iv) with decreasing strain. Figure 1(a) shows the stress-time dependence (left-hand scale). From the relaxation behavior on every single strain step one can clearly see that there are short relaxation times on a time scale up to about $2-3 \mathrm{~s}$,

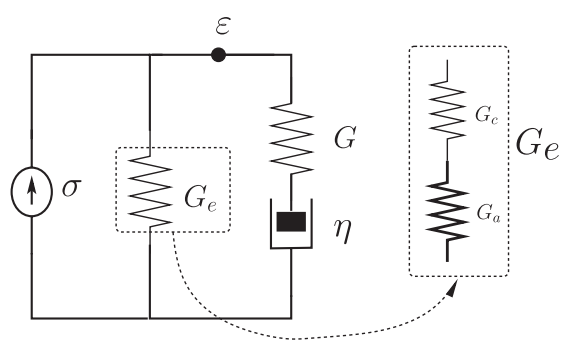

FIG. 2. Dashpot-spring diagram representation of the standard three-parameter Maxwell model in linear viscoelastic theory [21]. Springs $G_{e}, G$ represent the purely elastic and relaxing moduli, respectively, and $\eta$ stands for viscosity. Strain is symbolized by $\varepsilon$ and $\sigma$ stands for the measured stress. $G_{e}$ may be split up in elastic contributions $G_{c}$ and $G_{a}$ from crystalline and amorphous silk regions, respectively. slightly changing with the value of strain. There is an additional long relaxation time of the order of hours, which can be observed when an experiment is repeated a few days later and the fiber has almost recovered its initial length. On the time scale of our experiment, where we can approximate the strain increase with a constant strain rate [Fig. 1(a)], the responses are dominated by an intermediate characteristic relaxation time of about $10 \mathrm{~s}$. The stressstrain curves $\sigma(\varepsilon)$ [Fig. 1(b)] of the first and the second stretching experiment [(i), (iii)] show pronounced differences. The curves exhibit a bend (yield point) with a less steep curve afterwards. In the case of the unstretched sample (i) there is an extended plateau with little stress change. The prestretched sample (iii) reaches the same stress level at much smaller strain.

The prestretched sample surprisingly shows a behavior which allows a very simple phenomenological description in the context of linear viscoelastic theory. The schematic diagram of the model is shown in Fig. 2 in the form of a parallel connection, known as the standard three-parameter Maxwell model [21]. The model parameters $\left\{G_{e}, G, \eta\right\}$ are independent of the shape of the strain excitations. The equilibrium modulus $G_{e}$ defines the purely elastic behavior and is responsible for the asymptotic behavior at long times, the relaxing modulus $G$ and viscosity $\eta$ define the relaxation behavior. Secondary quantities of the model are the response time $\tau=\eta / G$ and the glassy stretch compliance (modulus) $G_{g}=G_{e}+G$. The latter defines the asymptotic behavior at small times and, thus, the initial modulus.

In this model, the response to a slope excitation of strain $\varepsilon(t)=\dot{\varepsilon_{0}} t$ is $[21,22]$

$$
\sigma(t) / \dot{\varepsilon}_{0} \equiv \tilde{\eta}(t)=G_{e} t+G \tau\left(1-e^{-(t / \tau)}\right),
$$

where $\dot{\varepsilon}_{0}=$ const is the strain rate and $\sigma(t)$ denotes stress. $\tilde{\eta}(t)$ is the time-dependent viscosity. The stress response for decreasing strain is given by

$$
\sigma(t)=\dot{\varepsilon}_{0}\left[\tilde{\eta}(t)-2 \tilde{\eta}\left(t-t_{\text {turn }}\right)\right],
$$

where $t_{\text {turn }}$ is the turning point of the triangle exitation. Equations (1) and (2) were fitted to the measured data, with fitting parameters $\left\{G_{e}, G, \tau\right\}\left(\dot{\varepsilon}_{0}=0.1 \%\right.$ is taken from a linear fit of the measured strain). This model fits the single fiber data of the prestretched sample with increasing (iii) and decreasing strain (iv) and of the unstretched sample in the initial state only for the direction of decreasing strain (ii) [Fig. 1(b), smooth curves). Obviously, on the time scale of the experiment, a single relaxation time of $\tau=10 \mathrm{~s}$ describes the data well. The equilibrium and the relaxing moduli are $G_{e}=5.1 \mathrm{GPa}, G=6.6 \mathrm{GPa}$, yielding $G_{g}=$ $11.7 \mathrm{GPa}$, in good agreement with [23].

It should be noted that the "yield point" of the prestretched fiber, which for similar systems is often interpreted as the result of strain-induced structural changes or even damage as a kind of "switch" for the mechanical 
properties of the amorphous matrix [24], originates in our model from the transition between the regimes where $G_{e}$ and $G$, respectively, dominate the response $\sigma(t)$ (1) of the material to strain.

The values $\left\{G_{e}, G, \tau\right\}$ have to be interpreted as average macroscopic material properties. As the fibroin crystals recover their initial $d$ spacing immediately after fiber rupture, as observed in experiments where fibers where stretched further, we know that the crystalline phase is purely elastic on the time scale of our measurements. One may thus interpret $G_{e}$ as the (elastic) property of the crystalline-amorphous composite and $\{G, \eta\}$ as parameters of the relaxation processes occurring only in the amorphous phase and possibly in the boundary region between the crystalline and the amorphous phases.

In a macroscopic model as the one presented here, neither the crystalline nor the amorphous elastic modulus are known a priori or from stretching measurements. In situ x-ray diffraction provides access to such information as it probes the lattice deformation of the fibroin crystals under tensile load. The protocol of the tensile testing experiment on fiber bundles was very similar to the single fibers experiment (Fig. 3, solid lines, right-hand scale): initial tensile deformation up to $\varepsilon \approx 6 \%$ (i), decreasing strain down to $\sigma=0$ (ii), strain increase of the prestretched sample up to previous maximum stress (iii). As compared to the single fiber experiment (Fig. 1), the general trends and integrated parameters are reproduced with bundles here; in particular, an initial modulus of about $11 \mathrm{GPa}$ is found. The differences in details such as the position of the yield point or the amount of plastic deformation are attributed to bundle effects [25].

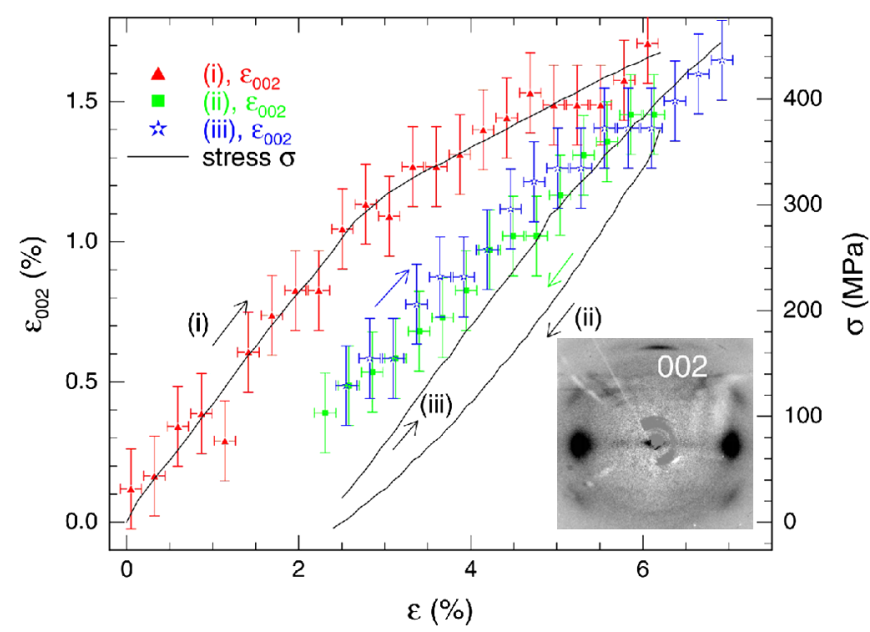

FIG. 3 (color online). Stress-strain curves of a bundle of silkworm silk fibers (solid lines, right-hand scale). As in Fig. 1(b), (i), (ii) denote data of the unstretched fibers (increasing and decreasing strain) whereas (iii) stands for the prestretched fiber bundle. The symbols give the strain $\varepsilon_{002}$ (left-hand scale) of the crystal lattice in axial direction, as determined from the meridional 002 reflections of the fiber diffraction diagram.
In the initial experiment on the unstretched sample, the crystal strain $\varepsilon_{002}$ (Fig. 3, triangles), determined from the 002 reflection [9], follows the shape of the stress-strain curve almost exactly. The appropriate scaling factor between the two scales in Fig. 3 was determined in a linear fit of $\sigma$ plotted against $\varepsilon_{002}$ (Fig. 4). There is no deviation from linear behavior even after the yield point at $\varepsilon_{002} \approx$ $1 \%$. The corresponding elastic crystal modulus is $G_{c}=$ $(26.5 \pm 0.8) \mathrm{GPa}$, in good agreement with previously published data ranging from 16 to $28 \mathrm{GPa}[9,16,26]$. Figures 3 and 4 thus provide strong evidence that the stress $\sigma$ is uniform over the fiber cross section; i.e., the fiber as a whole, the $\beta$-sheet crystals, and the amorphous regions carry the same load. The constant stress scenario has previously been suggested by Raman spectroscopic results [15]. It corresponds to a serial arrangement of crystalline and amorphous regions in the composite, known as the Reuss model in polymer physics [27]. The situation is schematically depicted in Fig. 2 where the elastic modulus $G_{e}$ is split into amorphous $\left(G_{a}\right)$ and crystalline $\left(G_{c}\right)$ elastances. It should be noted that those are in parallel to the elements $G$ and $\eta$ standing for the relaxation processes of the amorphous regions.

For a serial configuration of amorphous and crystalline elastances, the reciprocal value of the equilibrium modulus $G_{e}$ can be written as $1 / G_{e}=1 / G_{a}+1 / G_{c}$ [21]. $G_{e}$ and $G_{c}$ are already known from our experiments, so that $G_{a}=6.3 \mathrm{GPa}$ is calculated as the elastic modulus of the amorphous matrix, about 4 times smaller than $G_{c}$. Consequently, the amorphous matrix should account for most of the macroscopic fiber strain, which indeed has to be the case as can be seen in Fig. 3: at a macroscopic strain

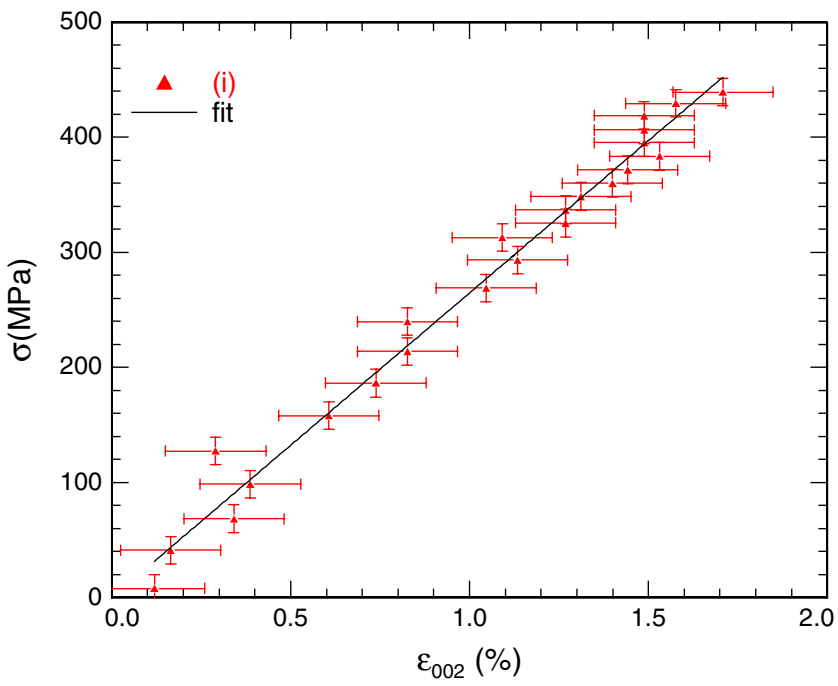

FIG. 4 (color online). Linear fit of the stress-strain curve of the fibroin crystals in silkworm silk [path (i) in Fig. 3]. The value for $\sigma$ was always taken from the stress-strain curve [solid line (i) in Fig. 3] at the strain $\varepsilon$ of the data points (triangles). A purely elastic modulus of $G_{c}=(26.5 \pm 0.8) \mathrm{GPa}$ is determined from the slope. 
of $\varepsilon=6 \%$, the crystal strain is only $\varepsilon_{002}=1.5 \%$, thus, a factor of 4 smaller.

The value of $G_{a}$ is in striking agreement with the relaxing modulus $G=6.6 \mathrm{GPa}$ from the viscoelastic model above, thus corroborating our microscopic interpretation of that model. Both $G_{a}$ and $G$ are mechanical parameters of the same amorphous silk material, describing its purely elastic and its viscoelastic response, respectively. In that picture, the crystals follow Hooke's law as perfect springs. They might thus be used as built-in "sensors" for stress in the composite material. The corresponding values of $\varepsilon_{002}$ for the cycle [(ii), (iii)] are shown in Fig. 3. Even though the general convex (ii) and concave (iii) shapes, respectively, of the stress-strain curves are reproduced, there is a remaining offset of about $0.4 \%$ at zero stress. The offset is too large to be explained by any unexpected changes of the x-ray scattering geometry (e.g., changed sample-todetector distance). If we take the additionally observed long relaxation time of the order of hours into account the crystal strain reflects the nonrelaxed stress in the amorphous silk regions.

Our simple viscoelastic model does not explain the stress-strain relationship of unstretched silk. From the details of the relaxation processes on the individual steps in the first stretching cycle (see Fig. 1) we deduce a rather sharp transition from purely elastic behavior up to the yield point to a fast relaxation process (on the plateau) with a typical relaxation time of about $1.5 \mathrm{~s}$. The plateau has been attributed to a hydrogen bond breaking process in the amorphous matrix $[8,13]$ or to a transition from a glassy to a rubberlike state [10]. The resulting flowlike extension with an activation barrier (yield point) of the amorphous protein chains would be consistent with the transition to the faster process we observe here, similar to a highly viscous polymer melt. In this context, amorphous silk protein might well be regarded as a rubber exhibiting entropy elasticity [28,29]. Our findings provide direct evidence for the proposed additional molecular mechanism leading to the pronounced plateau of unstretched silk stress-strain curves.

In conclusion, we clearly separated the mechanical properties of both the crystalline and the amorphous phase, as well as their interplay, of silk. A link between the macroscopic viscoelastic behavior and the mechanisms at the molecular length scale has been established. The $\beta$-sheet crystals do not only act as nodes in a disordered molecular network [8], but they are elastically deformed themselves and thus contribute to the extensibility of silk. Our new model is purely rheological and does not require any geometrical information (or assumptions) on the distribution of crystalline and amorphous phases, which an analysis in the framework of composite mechanics would do [14]. The model fully accounts for the semicrystalline morphology of silk and is also able to explain the mechani- cal properties of prestretched spider dragline silk, as we confirmed in a fit of data in [10].

We would like to thank I. Grotkopp (Kiel) and M. Dommach (HASYLAB) for technical support and W. Press and R. Berndt (Kiel) for valuable discussions. We gratefully acknowledge financial support by HASYLAB (II-04-051) and DFG (MU1673/3-1).

*mmueller@physik.uni-kiel.de

[1] C. Holland et al., Nat. Mater. 5, 870 (2006).

[2] Z. Shao and F. Vollrath, Nature (London) 418, 741 (2002).

[3] D. T. Grubb and L. W. Jelinski, Macromolecules 30, 2860 (1997).

[4] Y. Shen, M. A. Johnson, and D.C. Martin, Macromolecules 31, 8857 (1998).

[5] K. M. Parthasarathy et al., J. Appl. Polym. Sci. 59, 2049 (1996).

[6] D. Gersappe, Phys. Rev. Lett. 89, 058301 (2002).

[7] F. Junghans et al., Appl. Phys. A 82, 253 (2006).

[8] Y. Termonia, Macromolecules 27, 7378 (1994).

[9] A. Sinsawat et al., Polymer 43, 1323 (2002).

[10] D. Porter, F. Vollrath, and Z. Shao, Eur. Phys. J. E 16, 199 (2005).

[11] F. Vollrath and D. Porter, Appl. Phys. A 82, 205 (2006).

[12] H. Zhou and Y. Zhang, Phys. Rev. Lett. 94, 028104 (2005).

[13] Z. Shao and F. Vollrath, Polymer 40, 1799 (1999).

[14] J. Pérez-Riguero, C. Viney, J. Llorca, and M. Elices, Polymer 41, 8433 (2000).

[15] J. Sirichaisit et al., Biomacromolecules 4, 387 (2003).

[16] K. Nakamae, T. Nishino, and H. Ohkubo, Polymer 30, 1243 (1989).

[17] Degummed silkworm (Bombyx mori) silk (i.e., washed with warm distilled water to remove the sericin coating) was obtained from the Musée de la Soie, F-30170 StHippolyte-du-Fort, France. It consisted of fiber bundles of $(47 \pm 3.5) \mu \mathrm{m}$ in diameter containing 12 individual fibers (called brins) with a total cross section of (137 \pm 20) $\mu \mathrm{m}^{2}$.

[18] K. Kölln et al., J. Synchrotron Radiat. 12, 739 (2005).

[19] In view of the observed time scales for relaxation (extensively discussed below), the factor of 4 between the strain rates in the two experiments reported here is irrelevant.

[20] J. O. Warwicker, Acta Crystallogr. 7, 565 (1954).

[21] N. W. Tschoegl, The Phenomenological Theory of Linear Viscoelastic Behaviour (Springer-Verlag, Berlin, 1989).

[22] J.D. Ferry, Viscoelastic Properties of Polymers (Wiley, New York, 1980), 3rd ed.

[23] J. Pérez-Riguero et al., J. Appl. Polym. Sci. 70, 2439 (1998).

[24] J. Keckes et al., Nat. Mater. 2, 810 (2003).

[25] N. Pan et al., J. Mater. Sci. 32, 2677 (1997).

[26] A. Sinsawat et al., Polymer 44, 909 (2003).

[27] A. Reuss, Z. Angew. Math. Mech. 9, 49 (1929).

[28] J. M. Gosline, M. W. Denny, and M.E. DeMont, Nature (London) 309, 551 (1984).

[29] T. Seydel et al., Macromolecules 40, 1035 (2007). 